\title{
Research on the Path of Deep Fusion and Integration Development of Wuhan and Ezhou
}

\author{
Lijiang Zhao \\ School of Public Administration \\ Zhongnan University of Economics and Law \\ Wuhan, China 430073
}

\author{
Chengxiu Teng \\ School of Public Administration \\ Zhongnan University of Economics and Law \\ Wuhan, China 430073
}

\begin{abstract}
The integration development of Wuhan and Ezhou is a strategic task in Hubei Province. It is of great significance to enhance the primacy of provincial capital, form a new pattern of productivity allocation, drive the development of provincial economy and upgrade the competitiveness of provincial-level administrative regions. This paper discusses the path of deep integration development of Wuhan and Ezhou from the aspects of history, geography, politics and economy, and puts forward some suggestions on relevant management principles and policies.
\end{abstract}

Keywords-urban regional cooperation; integration development; path

\section{INTRODUCTION}

Exploring the path of leapfrog development in inland areas is a common issue for the vast areas (that is to say, 500 kilometers from the coastline) of China's hinterland. At the important time when China's economic development enters a new normal and we implement the Outline of the Development Plan for the Yangtze River Economic Belt, as the national first-class administrative region, the government of Hubei province has responsibilities to formulate corresponding policies, integrate resources and allocate power in the existing space. The consensus on the development pattern of "one provincial center of Wuhan and two provincial sub-centers of Xiangyang and Yichang" that has been formed in Hubei province will be promoted and realized by new measures under the new era conditions. Wuhan City, the provincial capital, is facing new opportunities and challenges. As a provincial government that mainly performs macro management functions, how does it provide new geographical space and policy support for the development of leading cities in Hubei Province?

\section{SOME ACHIEVEMENTS HAVE MADE IN THE}

\section{INTEGRATION DEVELOPMENT OF WUHAN AND EZHOU}

In the process of implementing the " $8+1$ " city circle strategy, the integration process of Wuhan and Ezhou has been greatly promoted. Wuhan and Ezhou are linked by mountains and rivers, and their economy is integrated and personnel relationship is close. They are closer than the other seven cities in the same urban circle. Ezhou City puts forward the work goals and ideas which are to take the lead in integrating into the Wuhan metropolitan area, speed up the urban integration of Wuhan and Hubei, rely on and undertake Wuhan. Ezhou City takes the initiative to revise the overall urban and rural plan. Ezhou's transportation infrastructure is connected to the traffic artery of Wuhan in an all-around and three-dimensional way. At present, there are 3 interconnected expressways including ShanghaiChengdu expressway, Wuhan-Ezhou expressway and Wugang expressway. In terms of market access, Wuhan East Lake Development Zone and Ezhou Gedian Development Zone try out market access cooperation, and enterprises registered in Ezhou can be named with "Wuhan".

\section{THE SPACE FOR IMPROVEMENT IN THE INTEGRATION DEVELOPMENT OF WUHAN AND EZHOU}

The degree of integration development of Wuhan and Ezhou is lower than that of central urban area of Wuhan, and suburban area of Wuhan including Jiangxia, Huangqi, Xinzhou and Caidian. The two cities still independently develop under the leadership of their respective administrative centers, but their cooperation has externalities, and there are huge transaction costs and repeated consideration of gains and losses in operation. There is a problem of insufficient integration in transportation, telecommunications, industrial gradient development, ecological protection, and public services. Wuhan's development stops at the intersection of Wuhan and Ezhou in many aspects.

There is room for adjustment in the integration of Wuhan and Ezhou in the system and mechanism. There are natural difficulties in the cooperation on the system between the two independent cities of Wuhan and Ezhou. Each has its own inconvenience and lacks substantive administrative communication channels. The long-term mechanism has not been established, and it still stays in the stage of "one case for one meeting". There are many contradictions between local interests formed under the current administrative system and regional economic integration, and there still exists discordance and excessive competition in industrial planning and investment attraction. Social undertakings cannot be deeply integrated, and the development achievements of metropolises have not yet benefited the surrounding cities. Therefore, we have to think about this issue from the provincial-level government. 
The ecological protection cooperation governance relationship of Liangzi Lake is fragile. Liangzi Lake is the second largest lake in Hubei province and the second water source site in Wuhan city. In the process of accelerating urbanization in Wuhan city, with the expansion of people's activity radius, Liangzi Lake has gradually changed from a suburb lake to an urban lake. Its ecological value is already higher than the production value, while the original management system has not changed, and the man-made ecological risk is increasing. The cooperative governance relationship is fragile, and overall management and protection are not effective enough, and the protection of ecological balance has not yet been put first.

There are barriers in the transportation and telecommunications markets in Wuhan and Ezhou. The subway that Ezhou people are looking forward to has not been connected to Ezhou, and the target of sharing the area code 027 by the fixed telephone has not been realized.

\section{ADMINISTRATIVE REGION ECONOMY DOMINATES THE PROCESS AND LEVEL OF PROVINCIAL AND MUNICIPAL ECONOMIC DEVELOPMENT}

China is a big country with unbalanced economic development, and the national unified market has not yet finally formed. Administrative region is not only a region for the state to carry out government affairs, but also a basic unit for allocating productive forces to promote economic development. Various local governments are important and active economic agents controlling a large amount of social resources, as well as the main enablers of economic development in administrative regions.

\section{A. Administrative Region Economy Dominates the Trend of China's Economic Development.}

At present, the actual situation is that the boundaries of administrative regions are hard to cross, and the integrated and regional development strategies are virtual. Although Hubei province has advantaged basic conditions, the pace of economic development is slower than that of coastal areas.

Based on the principle of administrative organization efficiency, there is always a dominant power center existing in any government. In general, a government is controlled by a single power center. The more decentralized power is, the more irresponsible it becomes. The more unified the power is, the more it will be guided by a responsible single power center. The formal cooperation system between the governments of Wuhan and Ezhou has not yet been established.

Over the ten years since the implementation of the Wuhan metropolitan area strategy, Wuhan's GDP reached 1341 billion yuan (RMB) in 2017. Now the trillion multiplication plan is being executed. The economic aggregate of each city in the eight cities, including Ezhou, Huangshi, Huanggang, Xiaogan, Xianning, and other cities, is only one-tenth of that of Wuhan, and their per capita GDP is only two-thirds of Wuhan. The phenomenon of industrial isomorphism is prominent in small and medium-sized cities in Wuhan metropolitan area. The metallurgy, textile and garment, and food processing and manufacturing industries exist in all small and medium-sized cities, and their competitiveness far exceeds the complementarity. Local governments often intercept the beneficial links in the market chain by administrative means to protect the weak competitive industries and defend the vested interests of the local. Therefore, market segmentation caused by the division of interests among localities has weakened the common interest base between local governments and reduced the trust between them, so that the real cooperation is difficult to achieve and the overall economic development of the Wuhan metropolitan area to the province is restricted.

\section{B. Mainland Provincial Capitals Are Competitive Areas Together with Coastal Regions}

The development gap between the coastal areas and the central and western regions is expanding. The inland provinces and cities are exploring the leap-forward development path, hoping to blaze a trail in the development of the economy. Sichuan, Henan, Hunan, Anhui, Shaanxi and other provinces have raised the primacy ratio of provincial capital, expanded development space, gathered energy and formed a new pattern of productivity allocation, in order to promote the economic development of the whole province and improve the competitiveness of provincial administrative regions. Sichuan province manages Jianyang on behalf of Chengdu City, and Anhui province abolishes the prefecture-level city of Chaohu and makes Hefei City bigger and stronger, and Hunan province creates ChangshaZhuzhou-Xiangtan urban circle, and they all follow the train of thought to drive the rapid development of provincial economy.

In 2017, Hubei province ranked seventh in terms of GDP among 31 provinces, autonomous regions and municipalities directly under the central government (excluding Hong Kong, Macao and Taiwan). Apart from the traditional economically strong provinces, including Guangdong, Jiangsu and Shandong, the ranking of other provinces may change at any time. The key to maintaining the ranking is which province has long-term and lasting institutional innovation. Hubei province steps up efforts to promote the integration of Wuhan metropolitan area, especially starting from Wuhan and Ezhou, which are the easiest part to be integrated, so as to form the most competitive growth core compared with coastal areas.

\section{The Integration of Wuhan and Ezhou Takes East Lake High-tech Development Zone as the Center and Wuhan as the Sub-Center in the Big Bay Area Along the Riverbank of the Yangtze River}

With the deepening of the integration of Wuhan and Ezhou, based on the principle of traditional Chinese administrative divisions with convenient mountain shapes, a relatively complete geographical space of ecology, productivity distribution, human flow and logistics has been formed in the big bay area along the Yangtze River of Ezhou $(80.1 \mathrm{~km})$ in Wuhan $(235.9 \mathrm{~km})$. 
Wuhan city develops from a single-nuclear city to a dualnuclear or multiple-nuclear city, forming a metropolis with the joint development of suburban and central cities. In the future, Ezhou will become the hinterland of rapid expansion and ecological protection space of East Lake High-Tech Development Zone bordering it, to provide a broader land, manpower, market and other resources for the industrial division and transfer of East Lake High-Tech Development Zone. It will become the growth pole in the east of Wuhan, and then the pattern and appearance of Wuhan will also undergo historic changes. East Lake High-Tech Development Zone with high-tech development as its main driving force and university cultural districts of Wuhan have been transformed from the edge area of the metropolis to the sub-center of the city.

\section{CONDITIONS AND BREAKTHROUGH FOR THE INTEGRATION DEVELOPMENT OF WUHAN AND EZHOU}

The integration development of Wuhan and Ezhou enhances their spillover advantages. As a national central city, Wuhan city shoulders a dual mission. In the face of new development opportunities, it will form a competitive development with other provincial capitals such as Chengdu, Xi'an, Changsha, Hefei, Nanjing, Zhengzhou, etc. Only by gaining new development momentum or generating new development space can it be possible to stand out from the crowd. Besides, as the leader of "one center and two subcenters" of Hubei province, Wuhan bears the major responsibility of leading the economic and social development of Hubei province. It will give play to the spillover effect of provincial capital city to integrate various production factors, gradually realizing balanced development within the province.

\section{A. Taking the Construction of Ezhou Airport as An Opportunity to Integrate Traffic of East Area of Hubei Province}

Ezhou airport is a major construction project during the 13th Five-Year Plan period in Hubei province. After completion, it will become the core base and national hub of S.F. Express. Its main function is to focus on freight and also give consideration to passenger. In the future, it will become a central freight hub covering the national air network, forming a dual-hub pattern of air passengers and freight within Wuhan metropolitan area. On the basis of deep integration with East Lake High-tech Zone, a multifunctional aviation metropolitan area including logistics, industry, commerce, tourism, leisure and vacation will be formed.

\section{B. Constructing Ecological Protection Zone in the Middle Part of Yangtze River and Liangzi Lake}

Wuhan and Ezhou are located in the middle reaches of the Yangtze River. Along the river, it is the east area of Hubei industry corridor dominated by metallurgy and building materials, gathering more than $30 \%$ of the pollution load in the province. We eliminate the abuses of the separate administration of each district, establish a unified environmental supervision and law enforcement agencies, straighten out the linkage mechanism of environmental protection, and improve the promotion of environmental protection in the middle reaches of the Yangtze River and the Liangzi Lake, which is conducive to the sustainable development of Wuhan metropolitan area and the establishment of national central cities as soon as possible.

Advantages create performance. The current passable ecological environment and abundant water resources are the biggest advantages for Hubei compared with Hebei and Henan provinces, as well as the greatest welfare of the people in the region. The integration of Wuhan and Ezhou will give play to an ecological advantage in a broader space. Protecting ecology of the Yangtze River is a national strategy, a regional strategy of Hubei Province and Wuhan City, and a desire of the people from the heart.

\section{Integrating Wuhan-Ezhou Industrial Cluster with East Lake High-tech Development Zone}

The producer of integration development of Wuhan and Ezhou can obtain a wide range of inputs from a wider range of industries, give full play to the scale economies effect in production, and reduce average production costs. In particular, East Lake High-Tech Development Zone, a hightech gathering place, will play its core and leading role. Along with the deep integration of Wuhan and Ezhou, the overall population, economy and geographical area of Wuhan City have been expanded. The advantages of intensive use of various resources in the region are more prominent, which is conducive to winning more support from the central government.

\section{The Integration of Wuhan and Ezhou Makes the History of Wuhan Richer and the Story of Hubei Longer}

Compared with Hankou, which has only risen for nearly 300 years, and Wuhan, whose raising period is less than 100 years, Ezhou is a place with rich history and stories. Located in the bay area along the Yangtze river, it has a unique regional culture and its administrative subordination has changed frequently. During the Shang Dynasty in 3000 years ago, it was called the E Country, and it was named E Town during the Warring States. During the Qin Dynasty, it was changed as E County and was affiliated to the south prefecture; in 1914, it was called Echeng County. In 1949, Echeng was managed by Daye sub-provincial administrative region; In 1965, Echeng County was zoned to Xianning Prefecture; In 1979, the county and city of Echeng was respectively set, and both were zoned to Huanggang region; In 1983, Echeng City and Echeng County were revoked, and Huangzhou Town of Huanggang County was incorporated and merged into Ezhou City directly under the central government; In 1986, Zuoling Town was subordinated to Hongshan District, Wuhan City; In 1987, Huangzhou District was put under the administration of Huanggang County. At present, the urban space of Ezhou City has been formed for only 30 years, and the frequency of administrative subordinate changes is rare in China. There must be laws to be found and the truth to be said. 
Ezhou is a famous historical city in Hubei province, as well as a place where emperors and nobles of successive dynasties and refined scholars stopped and lingered on. It has left countless treasures for Hubei culture and Chinese culture, which cannot be duplicated. Emperor Ying Zheng, King of Wu Sun Quan, Yue Fei, Zhu Yuanzhang, Ye Ting, Peng Dehuai, Wang Zhen, Li Xiannian, Mao Zedong, Li Peng, and others had all been to Ezhou, where the king duke general and minister had built their careers. The country also needs to be praised by scholars. For example, Qu Yuan, Li Bai, Meng Haoran, Cen Shen, Qian Qi, Lu Lun, Su Shi, Huang Tingjian, Zhang Lei, Qin Guan, Fan Chengda, and Lu You had famous works singing Ezhou or Wuchang in the world. Once the integration development of Wuhan and Ezhou is realized, the story of Ezhou is the story of Wuhan. For example, Dujiang Dam, Mount Qingcheng and Sanxingdui are part of the story of Chengdu; Lintong TerraCotta Warriors also becomes the story of Xi'an. This greatly enhances the richness and attractiveness of Wuhan metropolitan culture.

City is an important medium for human survival and development. It is not only the place where people live and work, but also the container of culture and the breeding place of new civilization. The integration development of Wuhan and Ezhou will build a bridge between history and future development under the scientific management of contemporary people, gradually realizing the rise of central Hubei.

\section{DIFFICULTIES IN THE INTEGRATION DEVELOPMENT OF WUHAN AND EZHOU AND ITS REALIZATION PATH}

The integration development of Wuhan and Ezhou is a strategic work in Hubei. It is a creative action and has farreaching significance. The so-called work is the conscious action of rational human beings. Unlike labor by means of external material, action does not require mediation of objects or things, and activities directly are carried out between people; it is committed to the establishment and maintenance of the political body and creates conditions for history. Actions establish connections between people, and build new public spaces, and are beneficial to the new structures of development of all parties.

\section{A. Options for Institutional Innovation: Administrative Trusteeship or Urban Council}

How to realize the integration development of Wuhan and Ezhou is a topic that the relevant stakeholders keep secret, as well as a difficult problem for the institutional innovation of Hubei province. It's been proven that under the economic conditions of the administrative region, the urban circle strategy and coordination mechanism are weak in promoting the integration development of large and mediumsized cities. The space expansion mode of Chengdu is simpler and more direct. The Xindu County, Wenjiang County and Shuangliu County were transformed from county into urban district. In 2015, the mayor of Chengdu, Tang Liangzhi, signed the document of Chengdu People's Government's Request for Related Matters of Handing Jianyang City as An Integrity to be trusteed by Chengdu City, to achieve substantive zoning changes in a gradual manner of trusteeship. The practices of brother provinces and cities can be used for reference in an innovative way. The key is whether the provincial and municipal leaders and the public can reach a consensus on the integration development of Wuhan and Ezhou, and form public opinion, to gradually realize the change of administrative division, that is, the integration development of Wuhan and Ezhou. At the same time, we can take the lead in creating a city council system and increase the voice of the suburbs in the metropolis.

\section{B. Restricting the Expansion of Low-density Cities and Protecting the Good Ecological Environment}

What kind of mechanism should be used to restrict the integration development of Wuhan and Ezhou and solve the problems of expansion of low-density cities, insufficient urban agglomeration and waste of land resources? The goals of integration development of Wuhan and Ezhou are not to expand the territory of Wuhan, but to integrate resources, optimize the industrial structure of the two cities, protect and optimize the ecological environment, and ultimately serve the people of the two cities.

\section{Balancing Interests and Realizing Equalization of Public Services of Wuhan and Ezhou}

What kind of mechanism should be used to achieve the equalization of public services and make the integration development of Wuhan and Ezhou benefit the people? Metropolis is the engine of economic and cultural development. Moreover, the cost of public services is relatively low. Wuhan City has the potential to provide public services for residents, and the limitations of the administrative districts in the past have not been better cultivated and released. Tongji Hospital and Hubei Provincial People's Hospital have established branches in the Optics Valley area. In terms of social security, transportation, telecommunications, finance, etc., Wuhan and Ezhou can also share. More refined, scientific and intensive management requirements have been put forward to the government.

About how to realize the change of ideas and the formation of consensus in the integration development of Wuhan and Ezhou, as long as we take actions in the integration development, there must be tension between stakeholders. The consensus on development and serving the people is the most important. A good way can be found to solve such problems in practice, provided we overcome the old traditional concepts and thoroughly reform our minds.

\section{CONCLUSION}

To realize the integration development of Wuhan and Ezhou, we need to change our ideas and reach a consensus. As long as we take actions in the integration development, there must be tension between stakeholders. The consensus on development and serving the people is the most important. A good way can be found to solve such problems in practice, provided we overcome the traditional old ideas, and do a good job in the improvement of administrative management 
system, public service coordination and interest balancing mechanism.

\section{REFERENCES}

[1] Wang Juan, Liu Sai. Study on the Coordinated Development of Comprehensive Transportation and Tourism Economy of Urban Agglomeration in the Middle Reaches of the Yangtze River [J]. On Economic Problems, 2018, Volume Missing (8): 111-118.

[2] Liu Xinyun, Zhang Jieke, Xing Yuan. Research on the Optimization of Regional Economic Structure in Wuhan [J]. Knowledge Economy, 2018, Volume Missing (14): 43-44.

[3] Zheng Jiangsong. Application of Shift-share Analysis Model for Industrial Structure and Industrial Competitiveness in Wuhan City Circle [J]. Journal of Hubei University of Technology, 2018, 33 (3): 25-30.

[4] Wang Haijun, Zhai Lijun, Liu Yanfang, et al. Urban Connection and Function in Wuhan Urban Agglomeration Based on MultiDimensional Urban Factor Flows [J]. Economic Geography, 2018, 38 (7): 50-58.

[5] Luo Nengsheng, Wang Yuze, Peng Yu, et al. Study on the Spatial Relationship of Eco-Efficiency of Urban Agglomeration in the Middle Yangtze River Basin and its Synergetic Promotion Mechanism [J]. Resources and Environment in the Yangtze Basin, 2018, 27 (7): 1444-1453.

[6] Ding Huang. Administration Science Third Edition [M]: Beijing: Capital University of Economics and Business Press, 2016.01: 89-100

[7] Huang Jun, Li Jun, Zhou Heng. The Construction of Evaluation System of the Function of National Central Cities: A Case Study of Wuhan [J]. Modern Urban Research, 2018, Volume Missing (5): 5564. 